\section{HIGH PRESSURE LIQUID CHROMATOGRAPHY OF THE MACROLIDE ANTIBIOTIC TYLOSIN}

\author{
Supapong BhuWapathanapun \\ and Peter Gray \\ School of Biological Technology, \\ University of New South Wales \\ Kensington 2033, N. S. W., Australia \\ (Received for publication March 28, 1977)
}

Tylosin is a member of the macrolide group of antibiotics and contains a sixteen-membered lactone ring. In common with many macrolide antibiotics it is a multi-component antibiotic, the structure of the major components being shown in Fig. 1. In order to study the regulation controlling the factor distribution in a fermentation it would be convenient to have an assay procedure that could separate and quantitate the various factors. Current assay techniques require the estimation of total tylosin factors by optical density measurements on a solvent extract of the broth followed by thin-layer chromatography to determine the various factors. High pressure liquid chromatography (HPLC) seemed to be a new technique that warranted investigation as an alternative assay technique.

Reports on the application of HPLC to the assay of antibiotics are rather limited and most are listed in a review by TsusI ${ }^{1)}$. For the macrolide antibiotics, the separation of erythromycin and anhydroerythromycin is mentioned in the above review and there has been a report ${ }^{2)}$ on the use of HPLC for the determination and factor

Fig. 1. Structures of the major tylosin factors.

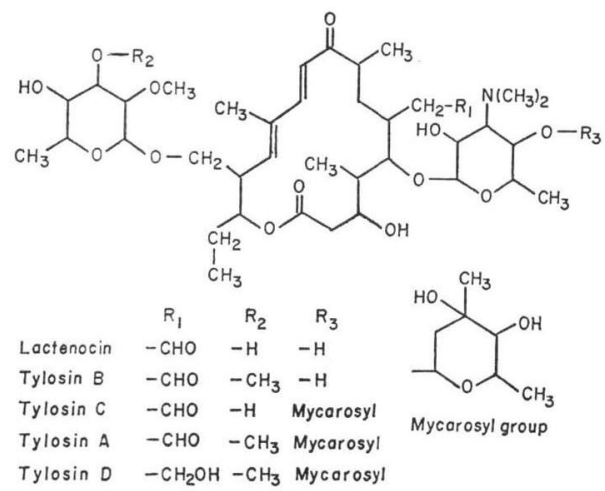

separation of leucomycin. The report by ŌMURA et $a l .{ }^{2)}$ also mentioned the use of HPLC for the determination of spiramycin, magnamycin, erythromycin and tylosin, however no details were given on the separation of the various tylosin factors. We have investigated the separation of tylosin factors using a Du Pont Model 841 HPLC. Initial work indicated that using filtered fermentation broth adversely affected both the resolution and column life, so subsequent work was carried out using a chloroform extract of the broth adjusted to $\mathrm{pH}$ 5.0. The Du Pont columns examined for the resolution of pure factors of tylosin A, B, C, D (and of tylosin present in fermentation broths) were ODS Permaphase and Sorbax SIL.

With the ODS-Permaphase column tylosin B could be separated from the other factors (Fig. 2) but tylosins $\mathrm{A}, \mathrm{C}, \mathrm{D}$ all had equivalent retention times and could not be separated even with a wide range of mobile phase compositions.

Fig. 2. High pressure liquid chromatography of tylosin complex.

Instrument: Du Pont 841, (254 nm detector, $4 \times$ $10^{-2}$ attenuation).

Column: Du Pont ODS Permaphase.

Column temperature: Ambient.

Column pressure: 1,200 psi.

Mobile phase: Methanol $75 \%$, water $25 \%$.

Flow rate: $1.0 \mathrm{ml} / \mathrm{min}$

Sample: Pure tylosin factors dissolved in chloroform, injection volume $5 \mu \mathrm{l}$.

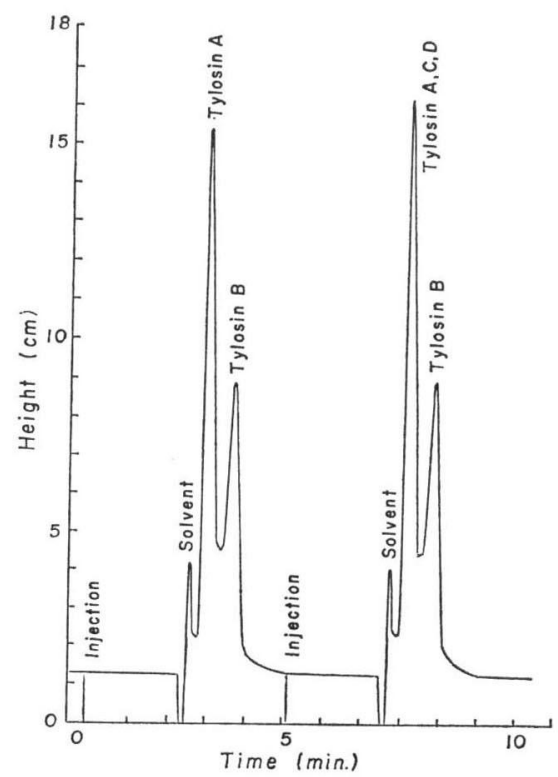


Fig. 3. High pressure liquid chromatography of tylosin complex.

Instrument: Du Pont 841, (254 nm detector, $4 \times 10^{-2}$ attenuation). Column: Du Pont Sorbax SIL. Column temperature: Ambient. Column Pressure: 1,200 psi.

Mobile phase: Chloroform $65 \%$, isopropanol $35 \%$. Flow rate: $1.0 \mathrm{ml} / \mathrm{min}$.

Sample: Pure tylosin factors dissolved in chloroform, injection volume $5 \mu 1$.

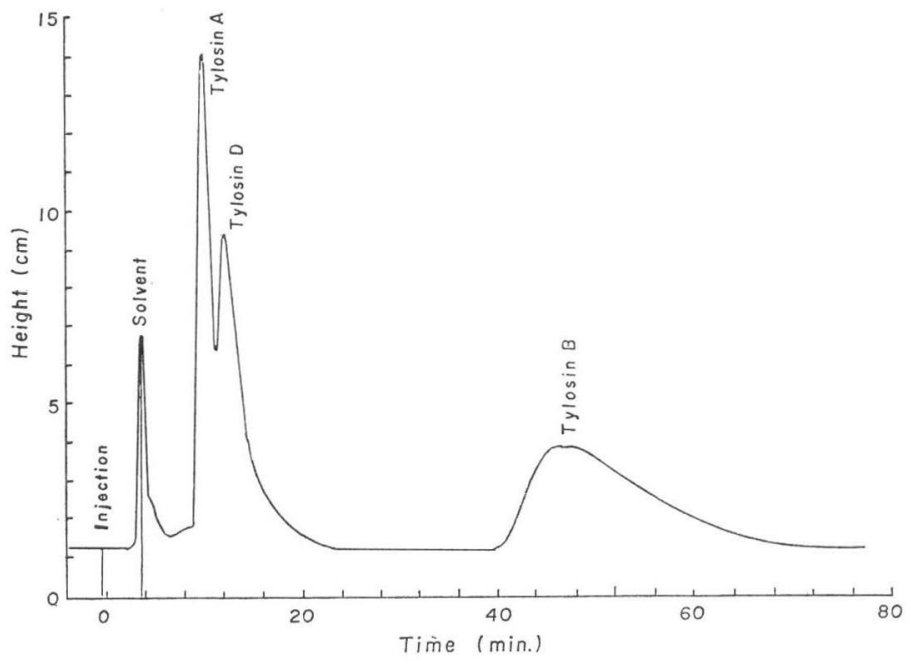

With the Sorbax SIL column and a mobile phase of $65 \%$ chloroform and $35 \%$ isopropanol the retention times of pure factors of tylosins A, C, D and B were 10.4, 12.4, 13.2 and 46 minutes respectively. The separation of a mixture of tylosins A, B and D is shown in Fig. 3. When tylosin $C$ was also present in mixtures, its peak could not be separated from that of tylosin D. If the isopropanol in the mobile phase was increased to $42.5 \%(57.5 \%$ chloroform $)$ then the tylosin $\mathrm{C}$ and tylosin $\mathrm{A}$ appeared as one peak separate from the tylosin D peak. Thus, by running each sample at the two mobile phase concentration, it was possible to quantitate the amount of tylosins A, C and D in mixtures containing the three factors.

With both columns a straight line relationship between peak height and concentration of tylosin A was obtained in the range $0.5 \sim 3.5 \mu \mathrm{g}$.
Using the Sorbax SIL column it was possible to separate and quantitate the four major tylosin factors. Further work using gradient elution might be able to further improve the separation and reduce the overall retention time.

\section{Acknowledgements}

The authors would like to thank Dr. F. M. Huber and Dr. P. Caltrider of Eli Lilly and Company for kindly supplying the pure tylosin factors.

\section{References}

1) TsusI, K.: High-pressure liquid chromatography of antibiotics. Methods of Enzymology, Vol. 43, pp. 300 319, Eds. S. P. Colowick \& N. O. KAPLAN, 1975

2) Ōmura, S.; Y. Suzuki, A. Nakagawa \& T. HATA: Fast liquid chromatography of macrolide antibiotics. J. Antibiotics 26: 794 796, 1973 